\title{
A Survey on Matching of Shoeprint with Reference Footwear in Forensic Study
}

\author{
Md. Al Mamun, Morium Akter, Mohammad Shorif Uddin* \\ Department of Computer Science and Engineering, Jahangirnagar University, Savar, Bangladesh \\ Email: al.mamun.1038@gmail.com, ecs_morium@yahoo.com, *shorifuddin@gmail.com
}

How to cite this paper: Al Mamun, Md., Akter, M. and Uddin, M.S. (2019) A Survey on Matching of Shoeprint with Reference Footwear in Forensic Study. Journal of Computer and Communications, 7, 19-26. https://doi.org/10.4236/jcc.2019.79002

Received: June 16, 2019

Accepted: September 1, 2019

Published: September 4, 2019

Copyright $\odot 2019$ by author(s) and Scientific Research Publishing Inc. This work is licensed under the Creative Commons Attribution International License (CC BY 4.0).

http://creativecommons.org/licenses/by/4.0/

\section{cc) (7) Open Access}

\begin{abstract}
Footwear impression marks on the outside surface of shoes are distinctive patterns and an important forensic clue often found at offense scenes. However, in many cases, the footwear mark is treated with improper evidence due to difficulties in visibility and understanding. This paper presents a thorough review of matching algorithms along with enhancement techniques of shoeprint in the forensic study. Finally, it shows some research directions.
\end{abstract}

\section{Keywords}

Forensic Study, Footwear, Shoeprint, Feature Extraction, Segmentation

\section{Introduction}

Footwear mark is eventually valuable evidence in forensic investigation. A footwear company develops footwear according to its customized design and contains size and shape difference with individual quality [1] [2] [3]. In forensic phenomena, the dimension of foot plays a fundamental role in constructing separate identity and the matching of footwear design characteristics [4] [5]. In addition to usual footwears, specialized footwears are used for various purposes such as physical therapy, sports, medicine and training, etc. [6] [7] [8] [9] [10]. However, for forensic evidence, an individual's shoeprint must be matched with the shoeprint stored in a database. The biometric footprint design has evolved from footprint recognition. To develop a database of footwear print images, it is necessary to collect the original shoeprint marks (edges) and the shoeprints on different surfaces. References [11]-[16] explain how a database of footwear images can be matched with shoeprint and measure their distortion rates. Here, the challenging issues: 1) shoeprint images are different on different ground surfaces due to variation of impressions; 2) walking, running or standing are different 
based on footwear design or conditions of outer edges of shoes, and, also wearer intentions. Figure 1 reflects full and partial footwear marks on different surfaces and Figure 2 presents known shoeprint marks which are indexed in a database as reference for matching [17]. For this, a preprocessing of shoeprint images (obtained in a crime scene) is essential before using a recognition algorithm. This paper discusses the present status of preprocessing and matching algorithms of shoeprint images along with a guideline for future research.

We have described the rest parts of the paper in the following two sections: Section 2 explains the footwear marks enhancement, detection and matching methodologies with critical comments and Section 3 presents the conclusion of the paper.

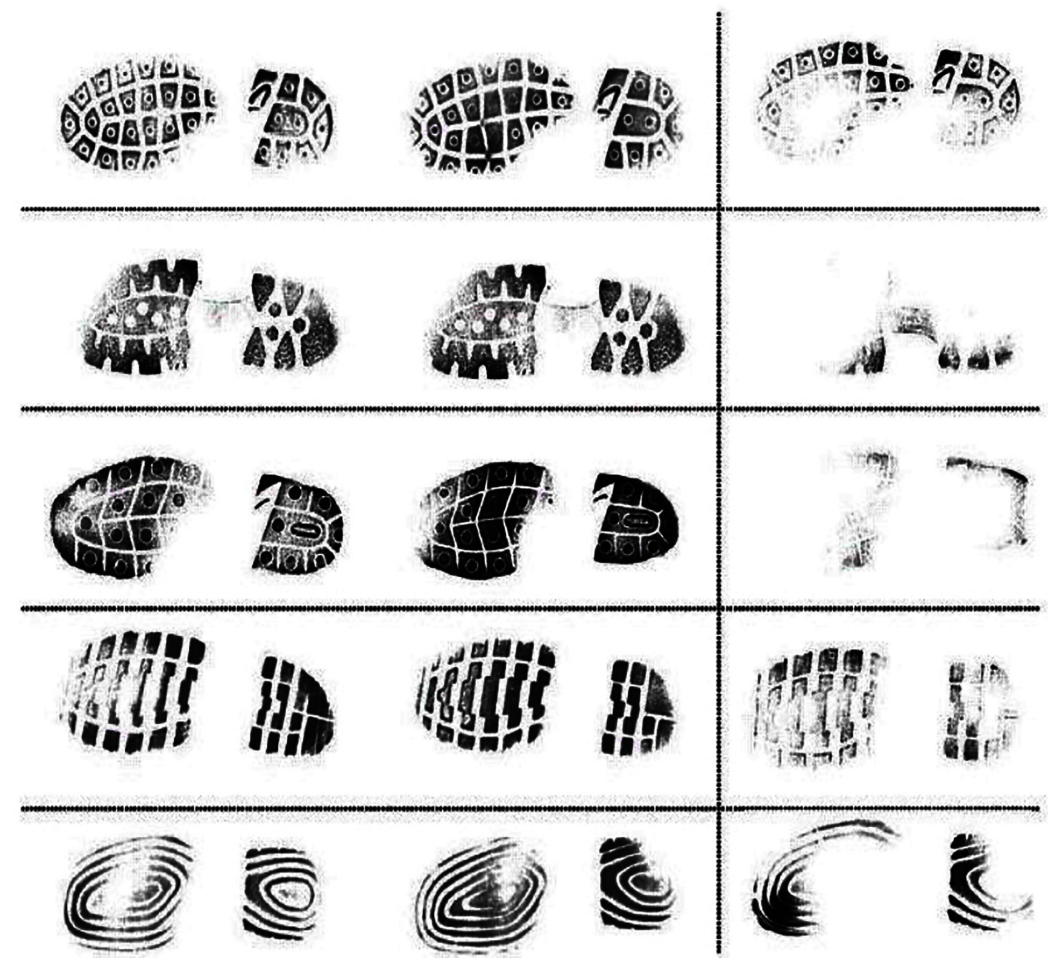

Figure 1. Example of five show shoeprint patterns. The left two columns show examples of images of full-shoeprints and the right column shows examples of images of partial shoeprints (image is taken from reference [17]).
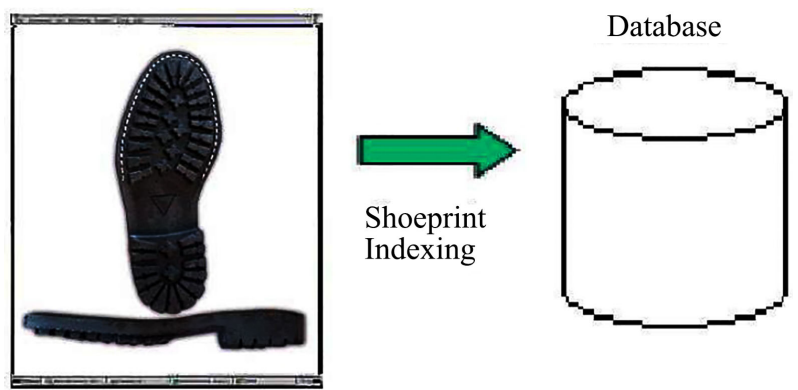

Figure 2. Known footwear print is scanned, processed and indexed in a database (image is taken from reference [17]). 


\section{Footwear Image Enhancement, Detection and Matching Methodologies with Critical Comments}

Usually, there are three forms of footwear evidences, such as footwear outsole impression, footwear insole impression and footwear trace evidence. The impressions of outsole are visible on doors or wall surfaces but not easily visible on tiles, concrete or carpet. For detection of footwear UV light and photography along with dust filters are used. In crime scenes, criminal creates footwear's outsole impression. The matching is done in between real crime scene shoeprint and the stored database shoeprints (that are designed by manufacturers).

Initial (raw) footwear evidence is recovered from shoeprint marks through a heterogeneous methodology:

- Impressions can be visualized with the help of natural or artificial lifting.

- Oblique, coaxial and polarized light is provided from artificial light sources.

- Electrostatic lifting is used to pick up dusty impressions.

- Fingerprint powders are used to build up latent impressions.

- Physical or chemical enhancement methods are used to form enhanced soft impressions.

These raw images are enhanced for clarity and matching with the reference shoeprint (obtained from the manufacturers) inside the database. For analysis of footwear evidence, an investigator needs to know different recognizable information such as the quantity of people in a crime scene, approximate height of them, activity (standing, walking, running or carrying a heavy load) of wearer when impression was made [6] [7] [8] [9] [10].

For matching, it is needed to establish a relation between crime scene impressions [11] [12] [13] [14] [15] with a specific piece of footwear. An investigator can determine the footwear impression uniquely if an absolute matching is found.

Existing shoeprint matching algorithms along with enhancement can be grossly classified into two groups: feature-based matching technique and probabilistic method. Some bench-marked methods are discussed below.

1) Feature-based matching technique

Kadam et al. [17] uses discrete Fourier transform to extract features for pattern matching in shoeprint-footwear. This method performs on 503 shoeprints. This method has a limitation as it did morphological operation without segmentation.

Pradeep et al. [18] presents a Radon transformation-based method that is invariant to rotation. This method shown in Figure 3 confirms good performance in shoe images with Gaussian white noise and salt-pepper noise. It can handle partial shoeprint images. The matching score for partial shoeprints is obtained about $95 \%$. It has lower computational time but is not appropriate for large database.

Xinnian et al. [19] proposed a fully automatic offense scene shoeprint retrieval algorithm that can be used to link crime scenes for finding out the brand of a 


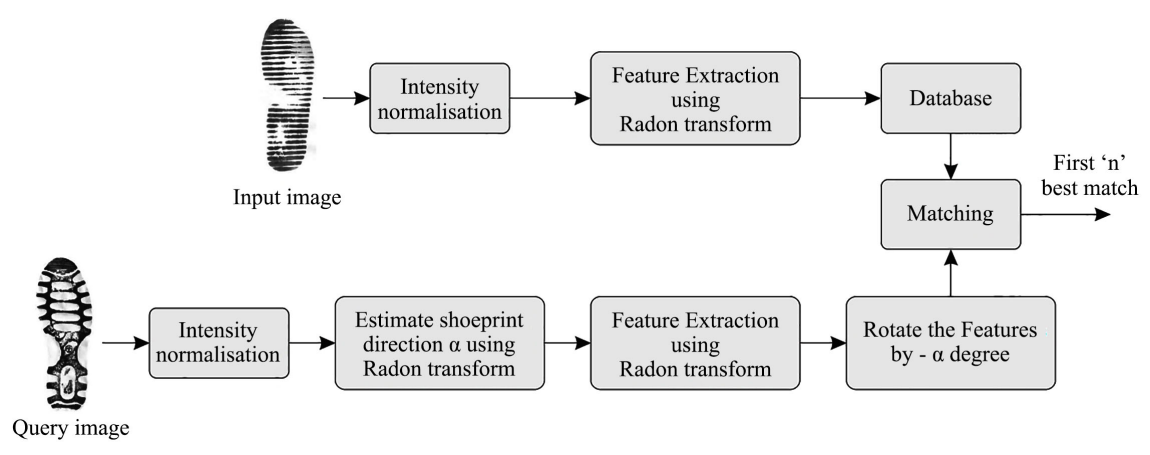

Figure 3. Proposed shoeprint matching system (image is taken from reference [18]).

shoe. At first, the shoeprint database is preprocessed to separate the shoeprint from background and then applied for feature extraction. Finally, correspondence measure is performed based on the extracted features. This method works on 210,000 shoeprints and the overall matching score is $90.87 \%$. However, this method is risky (due to false matching) and time-consuming.

Tang et al. [20] proposed a method that uses the elementary slopes in shoe features and retrieved the most analogous layout from a clustered database in noisy and blurry environments. This method did experimentation with only 100 real footwears and the obtained matching score is about $91 \%$.

Dong [21] described a method to extract features for the detection of shoeprint images. At first, footwear marks are extracted from the surfaces and then a special matching algorithm is applied for pre-matching of shoeprint image. Finally, the correlation coefficient is used for similarity measurement based on extracted features. It does not give good results in all situations.

Rathinavel and Arunugam [22] suggested a novel approach of 235 shoe print images based on discrete-cosine transform (DCT) coefficients. The similarity measurement is performed based on correlation coefficients. The peak similarity score is about $95 \%$. This method identified a combination of full shoeprint and partial shoeprint systems. For image matching, partial shoeprint resolves the memory constraint problem and speedup the computational time by reducing the number of DCT coefficients.

Maria and Nigel [23] suggested an automatic extraction and classification of footwear patterns using Harris affine transformation. The matching of shoeprint-footwear is obtained around $90 \%$ with an experimentation of 13,000 footwear patterns.

Dardi and Carrat [24] described a footwear detection system based on the Mahalanobis distance map. This method used processed footwear image rather than real image. In this analysis, a method based on luminance is applied to remove false border and edge in noisy footwear marks. It works on only 87 known shoeprints and the matching score is obtained around $91 \%$.

Rathinavel and Arunugam [25] proposed a shoeprint detection method using preprocessed (through enhancement and restoration) footwear marks. However, its performance is not up-to the mark. 
Khan and Tidke [26] showed the sketch of a method using Radon transformation without any detail experiment.

Xiangyang et al. [27] proposed a shoeprint detection system for forensic investigation using Gabor transform and histogram analysis. At first, Gabor transform extracts features and then integral histogram is applied for similarity measure. This method used only 195 footwear marks and the similarity score is found around 93\%. Fragmentation creates obstacle in extracting shoeprint features properly and consequently, matching score is not encouraging.

Manish and Pradeep [28] described an automatic shoeprint detection system for investigation of crime scenes. It uses Fourier features in finding matching score through Euclidian distance. It does not work well on large database.

Srihari and Tang [29] described a computational method for investigating footwear image. It uses 1000 known shoeprints and 50 real footwear marks and the obtained matching score is $92 \%$. It has depicted that computational method needs standard database for better matching of shoeprint-footwear.

Vaishal et al. [30] proposed a forensic investigation technique which uses a shoeprint image database. Gabor and Radon transforms are applied on routinely sorted query shoeprint images. It uses 40 footwear marks for analysis and the obtained similarity score is $90 \%$. This shoeprint matching technique needs larger database.

Rathinavel and Arumugam [31] proposed a system that converts images to gray scale and then histogram equalization is applied for image enhancement. Then inverse filtering is applied for image restoration and, finally, image segmentation is applied to index the shoeprint database storage. This method performs on only 50 footwear marks and the obtained matching score is somehow good.

Andres and Peter [32] suggested a foot biometric based approach. At first, edge detection and thresholding techniques are applied. Then texture segmentation and alteration invariant algorithms are applied for finding matching. This method experiments on only 160 footwear marks and obtained high matching score.

Chazal et al. [33] suggested a Fourier transformation-based automated processing of shoeprint images in forensic science. The method is not efficient, as it did not use segmentation, clustering and edge detection.

2) Probabilistic method

Vishnu and Srihari [34] proposed shoeprint extraction system by using probabilistic method rather than special thresholding and neural networks. This system used machine learning based probabilistic model for matching but could not provide a suitable result.

From the above analysis, most existing techniques have problems and at primitive stage. Therefore, it is preferable to concentrate on image enhancement, segmentation, feature extraction and pattern matching for improving the existing techniques for a reliable forensic investigation. A combination of feature-based and probabilistic methods may be an option for developing improved matching 
algorithms. In addition to normal probabilistic method, recently neural-based method (such as recurrent neural network (RNN), convolutional neural network (CNN), and deep learning) [35] shows promising results. Therefore, it may be a good direction to concentrate on RNN, CNN and deep learning for shoeprint recognition.

\section{Conclusion}

We presented the existing status of matching of shoeprint with reference footwear in the forensic study. Many researchers describe only preliminary stages of matching method; some researchers discuss a few matters and others proposed systems for matching images without proper enhancement and/or feature extraction algorithm. As a result, none of these approaches gives perfect results. Therefore, the paper addresses the shortcomings and recommends researchers to put emphasis on developing hybrid systems as well as neural-based approaches suitable for real-life applications.

\section{Conflicts of Interest}

The authors declare no conflicts of interest regarding the publication of this paper.

\section{References}

[1] Fatur, M.P., Novak, B., Alpina, D.D. and Dolinsek, S. (2007) Mass Customization in Footwear Industry: A Case Study. Proceedings of the 8 th International Management Conference, Slovenia, 20-24 November 2007, 1383-1389.

[2] Reyes, J., Aldas, D., Alvarez, K., García, M. and Ruíz, M. (2017) The Factory Physics for the Scheduling: Application to Footwear Industry. Proceedings of the 7 th International Conference on Simulation and Modeling Methodologies, Technologies and Applications, Madrid, 26-28 July 2017, 248-254. https://doi.org/10.5220/0006403402480254

[3] Witana, C.P., Feng, J.J. and Gooenetilleke, R.S. (2004) Dimensional Differences for Evaluating the Quality of Footwear Fit. Ergonomics, 47, 1301-1317.

http://www.tandf.co.uk/journals https://doi.org/10.1080/00140130410001712645

[4] Morio, C., Lake, M.J., Gueguen, N., Rao, G. and Baly, L. (2009) The Influence of Footwear on Foot Motion during Walking and Running. Journal of Biomechanics, 42, 2081-2088. https://doi.org/10.1016/j.jbiomech.2009.06.015

[5] Raju, G.M., Vijayanath, V. and Anitha, M.R. (2012) Estimation of Stature from Shoeprint Length While Walking in Females. Journal of Indian Academy of Forensic Medicine, 34, 288-291.

[6] Paradiso, J.A., Morris, S.J., Benbasat, A.Y. and Asmussen, E. (2004) Interactive Therapy with Instrumented Footwear. Proceedings CHI 2004, Vienna, 24-29 April 2004, 1341-1343. https://doi.org/10.1145/985921.986059

[7] Anbarasi, K. and Sharmila, L. (2013) Novel Footwear with Wireless Sensor Cater Using L.M.S Optimization Technique. International Journal of Advanced Research in Electronics and Communication Engineering, 2, 540-546.

[8] Whitting, J.W., Meir, R.A., Crowley-Manhattan, Z.J. and Holding, R.C. (2016) In- 
fluence of Footwear Type on Barbell Back Squat Using 50\%, 70\% and 90\% of One Repetition Maximum: A Biomechanical Analysis. Journal of Strength and Conditioning Research, 30, 1085-1092. https://doi.org/10.1519/JSC.0000000000001180

[9] Sinclair, J., Taylor, P.J., Edmundson, C.J., Brooks, D. and Hobbs, S.J. (2013) The Influence of Footwear Kinetic, Kinematic and Electromyographical Parameters on the Energy Requirements of Steady-State Running. Movement \& Sports Sciences, 80, 39-49. https://doi.org/10.1051/sm/2012025

[10] Sinclair, J., Taylor, P.J. and Atkins, S. (2015) Effects of New Military Footwear on Knee Loading during Running. Footwear Science, 1, 1-7.

[11] Holowka, N.B., Wallace, I.J. and Lieberman, D.E. (2018) Foot Strength and Stiffness Are Related to Footwear Use in a Comparison of Minimally- vs. Conventionally-Shod Populations. Scientific Reports, 8, Article No. 3679. https://doi.org/10.1038/s41598-018-21916-7

[12] Willems, C., Stassijns, G., Cornelis, W. and D’Aout, K. (2017) Biomechanical Implications of Walking with Indigenous Footwear. American Journal of Physical Anthropology, 162, 782-793. https://doi.org/10.1002/ajpa.23169

[13] Nagwanshi, K.K., Dubey, S. and Verma, T. (2018) Generic Biometric Footprint Recognition Framework for Personal Security. IJCEM International Journal of Computational Engineering \& Management, 21, 8-14.

[14] Khokher, R. and Singh, R.C. (2016) Footprint-Based Personal Recognition Using Scanning Technique. Indian Journal of Science and Technology, 44, 1-10. https://doi.org/10.17485/ijst/2016/v9i44/105167

[15] Smith, M.B. (2013) The Forensic Analysis of Footwear Impression Evidence. Forensic Science Communications, 11, Article ID: 233981.

https://www.ncjrs.gov/pdffiles1/nij/grants/233981.pdf

[16] Morgan, R.M., Freudiger-Bonzon, J., Nichols, K.H., Jellis, T., Dunkerley, S., Zelazowski, P. and Bull, P.A. (2009) The Forensic Analysis of Sediments Recovered from Footwear. In: Ritz, K., Dawson, L. and Miller, D., Eds., Criminal and Environmental Soil Forensics, Springer, Berlin, 253-269. https://doi.org/10.1007/978-1-4020-9204-6_16

[17] Kadam, A.B., Manza, R.R. and Kale, K.V. (2012) A Review: Analysis of Footwear Impression Evidence Collection \& Detection. International Journal of Machine Intelligence, 4, 410-413. https://doi.org/10.9735/0975-2927.4.2.410-413

[18] Patil, P.M., Deshmukh, M.P. and Kulkarni, J.V. (2012) Investigation of Shoeprints Using Radon Transform with Reduced Computational Complexity. Journal of Pattern Recognition Research, 7, 80-89. https://doi.org/10.13176/11.129

[19] Wang, X.N., Sun, H.H., Yu, Q. and Zhang, C. (2014) Automatic Shoeprint Retrieval Algorithm for Real Crime Scenes. In: Proceedings ACCV, Springer, Berlin, 399-413. https://doi.org/10.1007/978-3-319-16865-4_26

[20] Tang, Y., Srihari, S.N., Kasiviswanathan, H. and Corso, J.J. (2019) Footwear Print Retrieval System for Real Crime Scene Marks. In: IWCF 2010: Computational Forensics, Springer, Berlin, 88-100. https://doi.org/10.1007/978-3-642-19376-7_8

[21] Dong, Y.L. (2016) Matching Method of Partial Shoeprint Images Based on the PCA-SIFT Algorithm. International Journal of Engineering Research \& Science, 2, 167-171.

[22] Rathinavel, S. and Arumugam, S. (2011) Full Shoeprint Recognition Based on Passband DCT and Partial Shoeprint Identification Using Overlapped Lock Method for Degraded Images. International Journal of Computer Applications, 26, 16-21. https://doi.org/10.5120/3126-4301 
[23] Pavlou, M. and Allinson, N.M. (2006) Automatic Extraction and Classification of Footwear Patterns. Intelligent Data Engineering and Automated Learning, Burgos, 20-23 September 2006, 721-728. https://doi.org/10.1007/11875581_87

[24] Cervelli, F., Dardi, F. and Carrato, S. (2009) A Texture Recognition System of Real Shoe Marks Taken from Crime Scenes. ICIP 2009, Cairo, 7-10 November 2009. https://doi.org/10.1109/ICIP.2009.5413417

[25] Rathinavel, S. and Arumugam, S. (2009) Conversion of Commercial Shoeprint to Reference and Recovery of Images. International Journal of Image Processing, 3 , 164-169.

[26] Khan, M.A. and Tidke, S.M. (2013) Automated Processing of Shoeprint Images for Use in Forensic Science. International Journal of Advanced Research in Computer and Communication Engineering, 2, 4292-4294.

[27] Li, X.Y., Wu, M.H. and Shi, Z.P. (2014) The Retrieval of Shoeprint Based on the Integral Histogram of the Gabor Transform Domain. 8 th International Conference on Intelligent Information Processing, Hangzhou, 249-258. https://doi.org/10.1007/978-3-662-44980-6_28

[28] Deshmukh, M.P. and Patil, P.M. (2009) Automatic Shoeprint Matching System for Crime Scene Investigation. International Journal of Computing Science and Communication Technologies, 2, 281-287.

[29] Srihari, S.N. and Tang, Y. (2014) Computational Methods for the Analysis of Footwear Impression Evidence. In: Computational Intelligence in Digital Forensics. Forensic Investigation and Application, Springer, Berlin, 333-383.

https://doi.org/10.1007/978-3-319-05885-6_15

[30] Hire, V.R., Shaikh, F.I., Jadhav, J.B. and Joshi, M.V. (2012) A Novel Automated Shoeprint Matching Technique for Use as Forensic Evidence in Criminal Investigation. International Journal of Computer Applications, 48, 25-31. https://doi.org/10.5120/7338-0082

[31] Rathinavel, S. and Arumugam, S. (2009) Threshold-Based Indexing of Commercial Shoeprint to Create Reference and Recovery Images. Computer Science and Networking, 1, 59-62.

[32] Uhl, A. and Wild, P. (2008) Footprint-Based Biometric Verification. Journal of Electronic Imaging, 17, Article ID: 011016. https://doi.org/10.1117/1.2892674

[33] De Chazal, P., Member, J.F. and Reilly, R.B. (2005) Automated Processing of Shoeprint Images Based on the Fourier Transform for Use in Forensic Science. IEEE Transactions on Pattern Analysis and Machine Intelligence, 27, 341-350. https://doi.org/10.1109/TPAMI.2005.48

[34] Ramakrishnan, V. and Srihari, S. (2008) Extraction of Shoe-Print Patterns from Impression Evidence Using Conditional Random Fields. In: International Conference on Pattern Recognition, IEEE Computer Society Press, Tampa. https://doi.org/10.1109/ICPR.2008.4761881

[35] Shin, D., Lee, J., Lee, J. and Yoo, H.-J. (2017) 14.2 DNPU: An 8.1TOPS/W Reconfigurable CNN-RNN Processor for General-Purpose Deep Neural Networks. IEEE International Solid-State Circuits Conference, San Francisco, 5-9 February 2017. https://doi.org/10.1109/ISSCC.2017.7870350 